\title{
Dual adaptation and adaptive generalization of the human vestibulo-ocular reflex
}

\author{
ROBERT B. WELCH \\ NASA Ames Research Center, Moffett Field, Califormia \\ BRUCE BRIDGEMAN and JASON A.WILLIAMS \\ University of California, Santa Cruz, California \\ and \\ REGINA SEMMLER \\ University of Clausthal, Clausthal, Germany
}

\begin{abstract}
In two experiments, we examined the possibility that the human vestibulo-ocular reflex (VOR) is subject to dual adaptation (the ability to adapt to a sensory rearrangement more rapidly and/or more completely after repeated experience with it) and adaptive generalization (the ability to adapt more readily to a novel sensory rearrangement as a result of prior dual adaptation training). In Experiment 1 , the subjects actively turned the head during alternating exposure to a visual--vestibular rearrangement (target/head gain $=0.5$ ) and the normal situation (target/head gain $=0.0$ ). These conditions produced both adaptation and dual adaptation of the VOR but no evidence of adaptive generalization when tested with a target/head gain of 1.0. Experiment 2 , in which exposure to the 0.5 gain entailed externally controlled (i.e., passive) whole body rotation, resulted in VOR adaptation but no dual adaptation. As in Experiment 1 , no evidence of adaptive generalization was found.
\end{abstract}

The vestibulo-ocular reflex (VOR) is an open-loop compensatory system that is largely responsible for stabilizing the retinal image during rotational head movements. The eyes are driven by semicircular canal efferents in the direction opposite head rotation, with an eye/head gain of about 84 during passive rotation (Collewijn, Martins, \& Steinman, 1983). ${ }^{1}$ The VOR often operates in conjunction with other stabilization mechanisms - in particular, the visually induced optokinetic reflex, which allow ocular compensation for head movement to be nearly perfect (eye/head gain $=.94$; Collewijn et al., 1983).

Because the VOR is driven by the vestibular system, it can be isolated for study by removing all visual stimuli (i.e., by rotating the observer in the dark). Measured VOR gains vary widely among individuals, both human and monkey, and are known to be influenced by level of alertness and whether head rotation is active or passive (see, e.g., Jell, Stockwell, Turnispeed, \& Guedry, 1988).

\footnotetext{
The present research was supported by a Cooperative Agreement between the University of California, Santa Cruz, and the National Aeronautics and Space Administration (Project UPN 199-16-12-34). The authors thank Virginia Strain for her assistance in testing the subjects, as well as the three anonymous reviewers of our initial manuscript, whose constructive comments and criticisms led to the addition of an informative control condition and, in general, greatly strengthened this paper. The authors also thank Clifton Schor of the School of Optometry, University of California, Berkeley, for use of the rotating chair. Correspondence should be addressed to $R$. B. Welch, Mail Stop 239-11, NASA Ames Research Center, Moffett Field, CA 94035 (e-mail: rwelch @mail.arc.nasa.gov).
}

Active bodily rotation in the dark produces a VOR gain of about .96, as compared with the .84 gain reported by Collewijn et al. (1983) for passive rotation. The primary advantage of a vestibularly driven oculomotor system is its extremely rapid response time (i.e., the very short phase lag between head turning and compensatory eye movements). The vestibular signal stabilizes the visual field by greatly reducing the size of the error signal that remains to be addressed by the optokinetic system. An important reason for the shorter latency is the fact that vestibular receptors respond somewhat faster than visual receptors.

The perceptual concomitant of the VOR is the experience of an essentially stable visual world during head movements. When the VOR and other mechanisms fail to stabilize the visual field completely, as when one rapidly moves the head while viewing very small visual stimuli in an otherwise dark setting or when confronted with experimentally altered target-head gains, apparent concomitant motion is said to be occurring (Tietz \& Gogel, 1978).

\section{Adaptation of the Vestibulor-Ocular Reflex}

It has been known since Ronne (1923) that the primarily open-loop VOR system has a flexible aspect that allows for adjustments in gain (eye/head velocity) when confronted with visual-vestibular conflicts, also referred to as dysmetria. These perturbations can be either naturally or artificially induced. Thus, maturational changes in eye and head size, neural trauma, experimentally im- 
posed optical distortions, and altered vestibular environments all result in adaptive changes in the effective VOR gain that help to maintain optimum rotatory compensation.

Adaptation to altered VOR has been the subject of much research, primarily by experiments in which dysmetria has been introduced by systematically altering the visual stimulus relative to head position. For example, early research (e.g., Collewijn et al., 1983) often used magnifying goggles to produce the visual-vestibular conflict, since optical magnification necessitates changes in the amount of compensatory eye movement necessary to stabilize the visual environment during rotary head movements.

Recent technological advances have made it possible to create the necessary conditions for VOR adaptation by using a computer to generate visual stimuli that are coordinated with head movements. A typical protocol might entail causing a visual stimulus to move in tandem with head motion (target/head gain $=1.0$ ). This stabilization of the target, relative to head position, requires that the observer null the VOR completely if retinal stabilization of the image is to be achieved. Under experimental conditions, it is possible to produce substantial VOR gain adaptation in less than a minute of exposure to experimentally imposed dysmetria, especially if head movements are restricted to a single frequency (see, e.g., Melvill Jones, 1985).

Despite the demonstrable plasticity of the VOR gain, it is usually assumed to be a unimodal process. That is, it has been claimed that adaptive changes in gain are reflected in the neural structures that mediate the actual compensatory process and that there is, therefore, no unique neural structure where vestibular-ocular relations are stored besides those currently operating to control the adaptive process (Howard, 1982). Presumably, the system maintains its calibration of VOR gain either by measuring retinal slip (drift of the image on the retina) or by analyzing the associated oculomotor pursuit signal (Barnes, 1993).

\section{Dual Adaptation and Adaptive Generalization}

Most studies of adaptation to sensory rearrangement (e.g., prismatic displacement) entail one or perhaps two (temporally separated) periods of exposure to the distortion, each immediately followed by tests of negative aftereffects with the distorting medium removed and visual feedback precluded (see, e.g., Welch, 1978). However, Welch, Bridgeman, Anand, and Browman (1993) asked what would happen if subjects were required to alternate repeatedly between adapting to the sensory rearrangement and readapting to normal vision (or to another sensory rearrangement). They hypothesized that such a training regimen would eventually cause the adaptive process to become more efficient. That is, a given period of exposure to the distorted visual field might now be expected to produce adaptation that (1) reached asymptote sooner and/or (2) attained a greater magnitude than had been achieved as a result of the same period of exposure at the outset of alternation training. The results of the Welch et al. study confirmed this prediction for alternating adaptation and readaptation to rightward and leftward 15 -diopter $\left(8.6^{\circ}\right)$ prismatic visual displacement, and the authors dubbed the process dual adaptation.

In the same study, Welch et al. (1993) examined the question of whether dual adaptation training would also facilitate adaptation to a novel prism environment - that is, one that subjects had not previously experienced. This, too, they found, in the form of substantially increased adaptability to a 30 -diopter $\left(17.1^{\circ}\right)$ prismatic displacement. The authors referred to this phenomenon as adaptive generalization.

In summary, an attempt to produce dual adaptation (as well as adaptive generalization) requires that subjects undergo a schedule of repeated alternation between adaptation and readaptation to two (or more) different, mutually conflicting sensory environments. Logically, this capacity demands the presence of at least one cue (afferent or efferent) that reliably distinguishes the two environments. The alternating situations can entail either (1) a rearranged and a normal sensory environment or (2) two different sensory rearrangements, and the training program may be distributed over a period of hours or days. Assuming that such training leads to dual adaptation, a subsequent test for adaptive generalization is warranted. For this test, the subjects are allowed to adapt to an altered sensory environment that differs from both of the environments to which they have undergone dual adaptation training.

To the authors' knowledge, only Miles and Eighmy (1980) and Shelhamer, Robinson, and Tan (1992) have deliberately implemented a dual adaptation training regimen for VOR adaptation in primates. Miles and Eighmy, using rhesus monkeys as their subjects, failed to obtain evidence of dual adaptation and did not look for adaptive generalization. However, these investigators focused on long-term adaptation by having their subjects alternate between the different vestibular environments every 8 days, which might be too great an interval to produce dual adaptation. In contrast, the present experiments adapted human subjects for only a few minutes in each cycle.

Shelhamer et al. (1992) succeeded in producing dual adaptation with human subjects, although they, like Miles and Eighmy (1980), did not test for adaptive generalization. In the Shelhamer et al. study, subjects sat in a rotating chair and adapted to one form of altered target/head gain while their eyes were turned upward and another form with eyes turned downward. Postadaptation measures of the VOR revealed the appropriate switching of VOR gain as a function of the direction (up or down) in which the eyes were turned. Apparently, the efferent and/or afferent stimuli associated with eye direction served as the discriminative (or switching) cues for dual adaptation of the VOR in this experiment.

In the cat, Baker, Perlmutter, Peterson, Rude, and Robinson (1987) induced VOR dual adaptation by changing body orientation of the entire animal. This made oto- 
lith stimulation a discriminating cue. The result was a clear change in the direction of VOR, depending on orientation.

The primary aim of the present investigation was to reexamine the question of VOR dual adaptation and, more important, to determine whether the acquisition of such dual adaptation results in or entails adaptive generalization to a novel visual-vestibular environment. Also of interest was a comparison of active head rotation and passive whole body rotation as conditions for eliciting both of these adaptive processes.

Since VOR adaptation has been shown to occur even with diminutive visual stimuli (see, e.g., Post \& Lott, 1992), we made use of a point light source in a darkened room. This visual stimulus was coordinated with head movements, to produce the altered target/head gains. With a small target, visual pursuit should correct any errors in the VOR to enable accurate tracking; presumably, the pursuit signal detects VOR gain errors and drives adaptation. As frequency of sinusoidal oscillation decreases, pursuit becomes more important for driving eye movements and VOR less so. Our chosen oscillation rate was the highest practical rate that did not cause discomfort for the subjects over long exposure periods (Experiment 1) and that could be driven by our mechanical apparatus without distortion (Experiment 2). Finally, we used a 4min exposure period that, on the basis of the relevant research literature (e.g., Collewijn, Martins, \& Steinman, 1981), was expected to produce measurable adaptation.

Unlike the method of Barr, Schultheis, and Robinson (1976), our subjects performed the same task in the same way throughout the experiment. We used real visual targets in the exposure condition, rather than imagined targets, and always had subjects continue their exposure phase activity without interruption during the test phase in darkness.

\section{EXPERIMENT 1 Active Head Turning}

Method
Subjects
Sixteen male and female undergraduate and graduate students,
ranging in age from 18 to 22 , along with two of the authors, partic-
ipated in the Experiment on a voluntary basis. Most of the under-
graduate students served in partial fulfillment of a psychology
course requirement. All 18 subjects were either emmetropic or
slightly myopic, and all were able to focus the display successfully
without spectacles. All but 1 had normal oculomotor function,
whereas 1 was strabismic and was therefore tested monocularly, the
dominant eye unoccluded.

\section{Apparatus}

The subjects were seated before a hemicylindrical screen (viewing distance $=90 \mathrm{~cm}$ ) while a spot of red laser light was projected to a mirror and reflected onto the hemifield in front of them. This apparatus was shielded from the subjects' view. Head position was measured by means of a Polhemus Fastrak magnetic field emitter unit, in conjunction with a receiver mounted on a helmet that was tightly strapped to the subject's head. The mirror was coordinated with the head tracker by means of an IBM 486 computer, allowing concomitant movement of the laser spot at various softwarecontrolled target/head gains.

Eye position was monitored by paired infrared-sensitive photocells that were attached to the helmet directly below the subject's right eye. Error in the system was about $\pm 0.1^{\circ}$. The system was calibrated before each daily session by having the subjects look at targets to the left, center, and right of the centerline at the height of the adaptation target and equating the gains on the two sides. The computer converted (on line) the output of the eye monitor into degrees.

In early runs, we removed saccades from the raw data and found that this precaution made no difference to the results, presumably because these saccades were small and infrequent. Although saccades can be a serious problem when using continuous unidirectional vestibular or optokinetic stimulation (as in a rotating drum), this is not true for small-amplitude sinusoidal stimulation, especially with practiced subjects, as ours were.

\section{Design}

Ten subjects participated in one testing session each day for 5 consecutive days. (For reasons discussed later, this number was decreased to 8 subjects for the test of adaptive generalization). During each testing session, they were exposed alternately to the visualvestibular rearrangement (target/head gain $=0.5$ ) and the normal visual-vestibular relationship (target/head gain $=0.0$ ). Immediately after the last of these dual adaptation training sessions (Day 5), they were tested for possible adaptive generalization by being exposed and allowed to adapt to an altered visual-vestibular arrangement (target/head gain $=1.0$ ) that differed from the one they had experienced during the preceding dual adaptation training sessions.

Eight control subjects underwent only one testing session, during which they were exposed to the same adaptive generalization stimulus that the experimental group saw. It might be argued that this control group should have undergone the same 5 consecutive days of testing as our experimental group before finally being cxposed to the adaptive generalization stimulus. Presumably, subjects in such a proposed control condition would be exposed to a stationary visual stimulus on each day until finally experiencing the adaptive generalization stimulus at the very end. However, because such a regimen during the first 4 testing days would merely have reinforced what these subjects would have been experiencing in their everyday life (i.e., a stationary visual world), it seems rather unlikely that such a control group would be preferable over the group that we chose to use.

\section{Procedure}

Baseline measures. Each daily dual adaptation training session began with baseline measures of the VOR gain following exposure in the experimental apparatus to a target/head gain of 0.0 . The subjects rotated the head from side to side to the beat of a metronome that was set at $0.45 \mathrm{~Hz}$ (i.e., one complete head-turning cycle every $2.2 \mathrm{sec}$ ) while fixating the stationary dot of light on the screen in the otherwise dark testing room. During this $25-\mathrm{sec}$ period, eye movements were monitored, although they were not recorded because they were theoretically uninformative. The subjects simply tracked the target with a combination of pursuit and VOR-driven movements. Tracking was nearly perfect at the frequencies we used. Then, while subjects continued to oscillate the head, a computercontrolled shutter extinguished the visual stimulus, and eye movements were recorded in the dark, sampled at $43 \mathrm{~Hz}$ for $5 \mathrm{sec}$. These were the VOR (eye/head gain) measures. The subjects had been instructed previously that during these measures they were to fixate an imagined point in the dark, straight ahead of them. Immediately after the 5-sec VOR-recording interval, the visual target reappeared 
as the subjects continued to engage in the head movements for the next cycle. Four such determinations were made and averaged to arrive at a measure of daily baseline gain.

Dual adaptation training. The subjects in the experimental group underwent four 55 -sec periods of visual-vestibular conflict, during which the target/head gain was set at 0.5 . Thus, the spot moved in the same direction as that in which the head was turning, but by half as much. Each period was followed by a 5-sec VOR gain recording interval in the dark, using the procedure described above. This adaptation period was followed by a readaptation phase consisting of four 55-sec periods of exposure to a stationary visual target (target/head gain $=0.0$ ), each followed by a 5 -sec VOR recording interval in the dark. This procedure met the requirements for dual adaptation training, since subjects were alternated repeatedly between exposure (and, hopefully, adaptation) to an altered sensorimotor environment and exposure (readaptation) to the normal environment.

Test for Adaptive Generalization. The possibility that dual adaptation had resulted in adaptive generalization was tested by following the readaptation phase of the final dual adaptation training session (Day 5) with a period of exposure to a target/head gain of 1.0 (i.e., target moves in the same direction as the head and by the same amount). In order to discharge any adaptation that might have remained from the preceding dual adaptation phase of that testing session, the subjects were instructed to walk around the now-illuminated laboratory before exposure to the adaptive generalization stimulus commenced.
The presence of adaptive generalization would be confirmed by finding more rapid and/or complete adaptation for the experimental group than for the control group, the latter being exposed to this visual-vestibular rearrangement but not having previously undergone dual adaptation training. Following calibrations, the control subjects underwent a baseline measure with a target/head gain of 0.0 , in the same manner as the experimental group. This procedure gave the control subjects practice with exposure to the experimental protocols and procedures.

\section{Data Analysis}

The VOR was assessed in terms of eye/head gains that were defined as mean velocity ratios (i.e., the ratio of average eye velocity to average head velocity, measured as root mean square [RMS] amplitudes of the sinusoidal movements). The four baseline eye/head gains for a given day were averaged to produce an overall baseline for that day. Subsequent gain measures were normalized by multiplying them by the reciprocal of this baseline measure. Thus, adaptation was measured relative to a given subject's initial VOR gain on that day. The normalized gains for all conditions were combined across the 10 experimental subjects, yielding 20 data points for the four trials within each of the 5 days.

Since the baseline VOR gain is a calibration that, by definition, has no variance, the statistical analysis of VOR adaptation did not include it. Therefore, the first step was to determine, by means of a $t$ test, whether adaptation at the 1 st min was statistically different from zero. Subsequent differences among all of the exposure tests

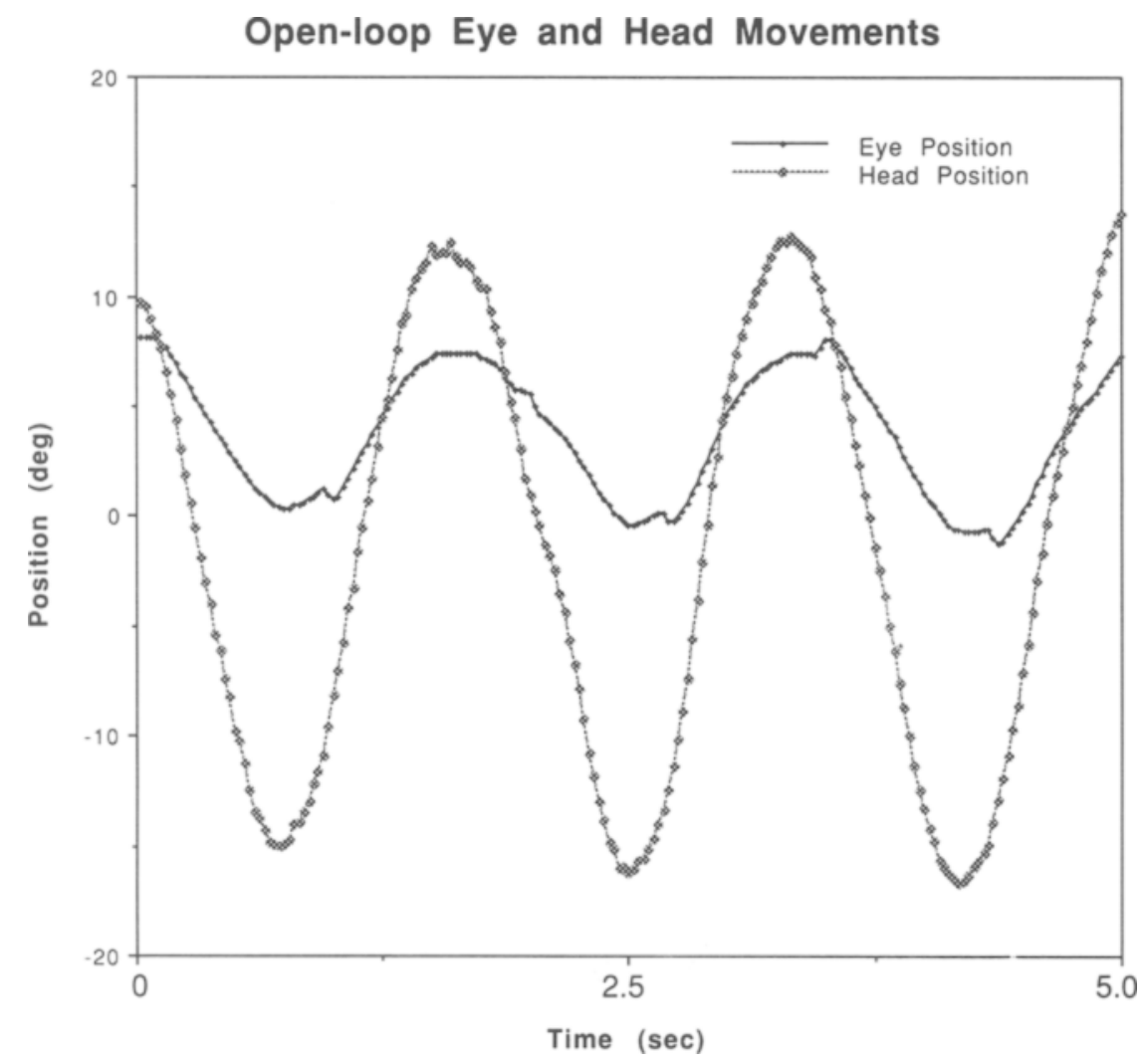

Figure 1. Eye and head position records during a single trial for a single subject in the baseline condition. Head record is inverted to make phase relationships clearer. Each point on the records indicates a data sample. Typically, very small saccades $\left(<0.5^{\circ}\right)$ occur just after the time of maximum eye acceleration when VOR reverses direction, even though there is no visual feedback during data recordings. This indicates that the VOR compensates both velocity and position. 
(excluding the baseline) were analyzed by means of analyses of variance (ANOVAs)

\section{Results}

An example of eye and head positions during a single trial of the open-loop test (baseline) condition is shown in Figure 1.

\section{Adaptation and Dual Adaptation}

Adaptation to the 0.5 target/head gain would be revealed by a reduction of VOR (eye/head) gain for one or more of the four tests of a given session, relative to the baseline (zero) gain. Naturally, if the VOR gain reached 0.5 , adaptation would be complete. No adaptation was evident at any of the tests on Day $1[t(9)=1.20, p=.260]$ but did occur for some or all tests on subsequent days, with the adaptation curves becoming progressively steeper over successive testing days. This latter fact is congruent with the presence of dual adaptation. Closer examination of the data revealed that virtually all of the adaptation for a given session occurred in the $1 \mathrm{st}$ min of exposure. A $t$ test comparing the baseline with the $1 \mathrm{st}$ min was statistically significant $[t(9)=2.64, p=.027]$. Nevertheless, adaptation fell far short of the theoretical maximum (VOR gain $=0.5$ ), not an uncommon finding in studies of adaptation to sensory rearrangement (see, e.g., Welch, 1978). A two-way ANOVA was performed, with day and exposure minute (excluding baseline) as factors and VOR gain as the dependent variable. A main effect for day (see Figure 2) was found $[F(4,165)=3.4$, $p=.01]$, confirming that dual adaptation had occurred. It is clear that asymptotic adaptation to the altered visualvestibular conditions increased with repeated exposure, in that the Day 5 VOR gain represents a reduction of nearly $14 \%$, relative to Day 1 . No main effect was obtained for either exposure minute $[F(3,166)=0.22, p=.88]$ or the interaction $[F(12,150)=0.34, p=.98]$.

\section{Adaptive generalization}

The results pertaining to possible adaptive generalization are summarized in Figure 3. Unfortunately, because of technical difficulties, 2 of the 10 dual adaptation subjects could not be tested, leaving 8 subjects in this group. As a consequence, the adaptive generalization control group was limited to 8 subjects as well. According to $t$ tests, 1 st-min adaptation was significantly greater than zero for both experimental $[t(7)=8.70, p<.001]$ and control $[t(7)=2.81, p=.026]$ groups. However, a group $\times$ exposure minute (1-4) ANOVA failed to produce a significant main effect for group [experimental/control, $F(1,61)=0.50, p=.48]$, implying the absence of adaptive generalization. In addition, no significant main effect was obtained for exposure minute $[F(3,59)=0.65$, $p=.59]$ or for the interaction $[F(3,55)=0.20, p=.90]$. This outcome cannot be due to a weak effect being masked by inadequate statistical power, since the subjects in the control group actually adapted (nonsignificantly) faster than did the subjects in the experimental group. Thus, no evidence for adaptive generalization of the human VOR was obtained.

\section{Discussion}

The present finding of dual adaptation in the absence of adaptive generalization contrasts with the results of the prism adaptation study by Welch et al. (1993), who found evidence for both of these adaptive processes, and of the Experiment by Miles and Eighmy (1980), who found neither for the VOR in monkeys.

In order to provide better control for the amplitude of head movements and to eliminate any possible influence of the vestibulocolic (neck) reflex, a second experiment was implemented, in which we examined both dual adaptation and adaptive generalization when the entire body was rotated by external means (passive rotation). Another important reason for wanting to determine the effects of

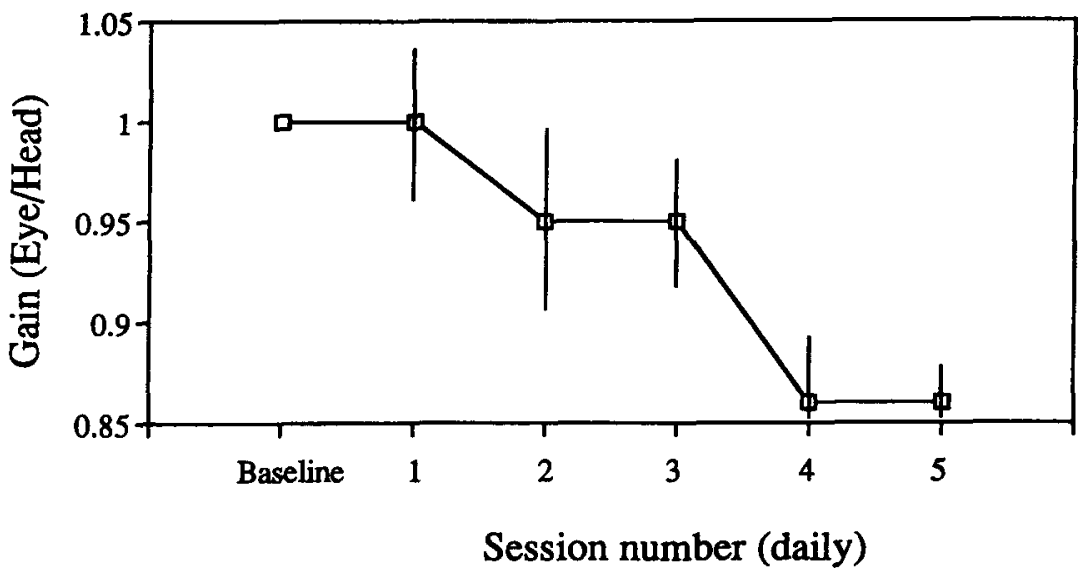

Figure 2. Experiment 1: Visual-vestibular gain (averaged over four exposure times) as a function of sessions/day. Baseline represents a normalized average of four preadaptation tests. Subsequent gains are evaluated relative to this measure. Errors bars represent the standard error of the mean. 


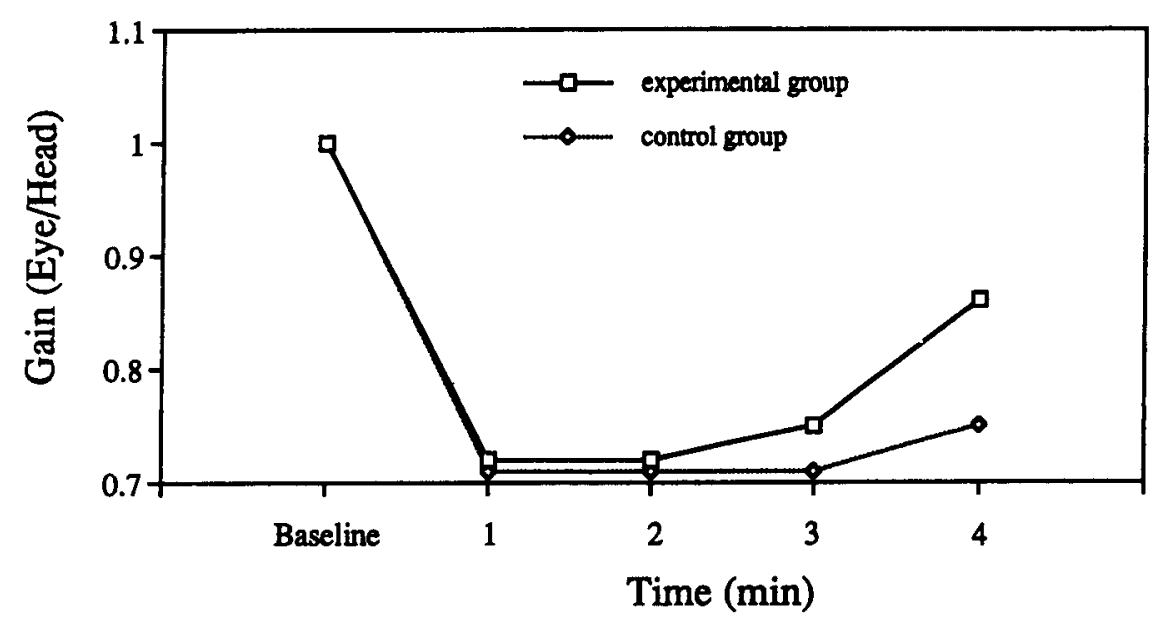

Figure 3. Experiment 1: Visual-vestibular gain (averaged over four trials) as a function of exposure time for experimental (dual-adapted) and control groups. Baseline represents a normalized average of four preadaptation tests. Subsequent gains are evaluated relative to this measure.

passive bodily rotation is the existence of many real-life situations of externally driven bodily movements in which VOR adaptation is likely to occur. Examples include space and airplane flight, as well as certain nautical environments. In short, it is important to determine whether the effects of active rotation are also found in the passive situation. An attempt was made to keep the experimental conditions of Experiment 2 as similar as possible to those of Experiment 1, except for the introduction of passive oscillation by a mechanical chair.

\section{EXPERIMENT 2 \\ Passive Whole Body Turning}

Method

\section{Subjects}

Eight male and female psychology undergraduate and graduate students, aged 18-22, formed the dual-adapted (experimental) group, while a second set of 8 subjects made up the non-dualadapted (control) group. As in Experiment 1, all subjects had either emmetropic or slightly myopic vision. The latter were tested either while wearing corrective lenses or in their myopic state, with spectacles removed.

\section{Apparatus}

The apparatus was identical to that used in Experiment 1, with the exception that the subject was seated in a chair that oscillated sinusoidally about a vertical axis at the same frequency $(0.45 \mathrm{~Hz})$ as in Experiment 1 and at a peak-to-peak amplitude of $16^{\circ}$.

\section{Procedure}

Aside from the use of passive rotation, the procedure was identical to that of Experiment 1 . As before, the normalized VOR gains of the 8 subjects were averaged, yielding 20 conditions - four trials within each of the 5 consecutive days.

\section{Results}

\section{Adaptation and Dual Adaptation}

Adaptation was evident on the 1 st testing day, collapsed across exposure minute $[t(7)=2.43, p=.045]$, and after the $1 \mathrm{st}$ exposure min, collapsed across days $[t(7)=4.92, p=.002]$. For subsequent exposure minutes, a day $\times$ exposure minute (1-4) ANOVA was performed, with VOR gain serving as the dependent variable. As in Experiment 1, this test failed to produce a significant main effect for exposure minute $[F(3,134)=$

$4, p=.59]$. This outcome, combined with the 1 stmin results presented above, indicates that most or all of the VOR adaptation occurred during the 1 st min of exposure. Further, although there seemed to be a trend toward more complete adaptation over days (see Figure 4), this also was not statistically significant $[F(4,133)=$ $1.4, p=.26]$ after the 1 st day. Finally, there was no interaction $[F(12,118)=0.73, p=.72]$.

\section{Adaptive Generalization}

Although a $t$ test failed to reveal significant adaptation in the $1 \mathrm{st} \min$ for the experimental group $[t(7)=$ $1.25, p=.25]$ and only marginal adaptation for the control group $[t(7)=2.23, p=.06]$, the results for the last min of exposure were statistically significant for both groups $[t(7)=2.99, p=.020$, and $t(7)=3.32, p=013$, for experimental and control groups, respectively]. An exposure minute $(1-4) \times$ group (experimental/control) ANOVA (see Figure 5) once again failed to produce a main effect for either exposure minute $[F(3,59)=1.0$, $p=.39]$ or group $[F(1,61)=0.63, p=.43]$. The trial $\times$ group interaction was also nonsignificant $[F(3,55)=$ $0.17, p=.91]$, although, as before, it appears that the control group might actually have adapted more than the experimental group.

\section{Discussion}

This experiment demonstrated that passive whole body rotation is an adequate condition for producing VOR adaptation, replicating the results of many earlier studies (e.g., Melvill Jones, 1985). Indeed, a comparison of the 


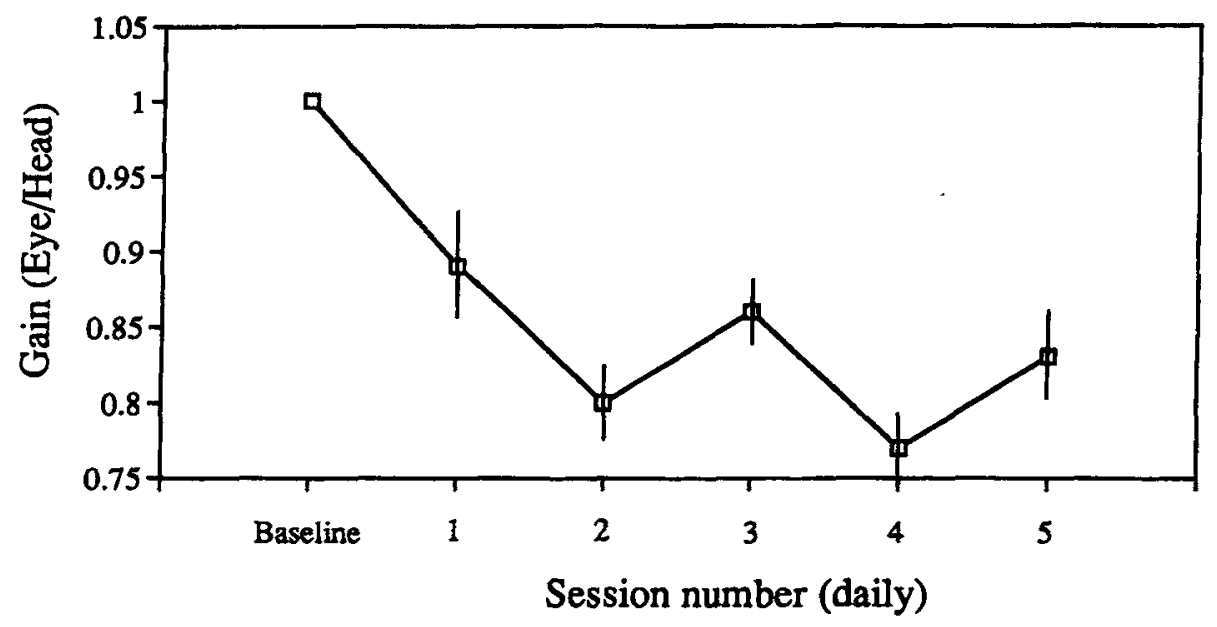

Figure 4. Experiment 2: Visual-vestibular gain, collapsed over four trials, as a function of sessions/day. Baseline represents a normalized average of four preadaptation tests. Subsequent gains are evaluated relative to this measure. Errors bars represent the standard error of the mean.

results of the two experiments suggests that, if anything, adaptation was greater for passive than for active movement. This possibility is examined more closely in the next section. In contrast to the results of Experiment 1, however, only a statistically nonsignificant trend in the direction of dual adaptation was observed in this experiment. Consequently, the failure to obtain adaptive generalization was not unexpected, since its occurrence is predicated on the presence of dual adaptation.

\section{Comparison of Active and Passive Conditions}

Inasmuch as the procedures for Experiments 1 and 2 were virtually identical, except for the active/passive difference in the way bodily rotation was effected, a statistical comparison between them may be considered legitimate and useful. With respect to adaptation, a day $\times$ rotation (active/passive) ANOVA produced a significant main effect for rotation $[F(1,80)=6.65, p=.01]$, the advantage going to passive rotation, but not for day $[F(4,77)=1.94, p<.05]$ or the interaction $[F(4,77)=$ $0.33, p>.05]$. Since the effect of exposure minute had not been statistically significant in either experiment, this factor was not reexamined here. A rotation (active/ passive) $\times$ group (experimental/control) ANOVA with respect to the 1.0 target/head gain adaptive generalization test (compare Figures 2 and 4 ) failed to find statistical significance for rotation $[F(1,31)=0.42, p>.05]$, group $[F(1,31)=0.33, p>.05]$, or the interaction $[F(1,31)=$ $0.01, p>.05]$.

The finding of greater adaptation for passive than for active rotation is surprising, since it contradicts a great deal of previous research (see Welch, 1978, pp. 21-24).

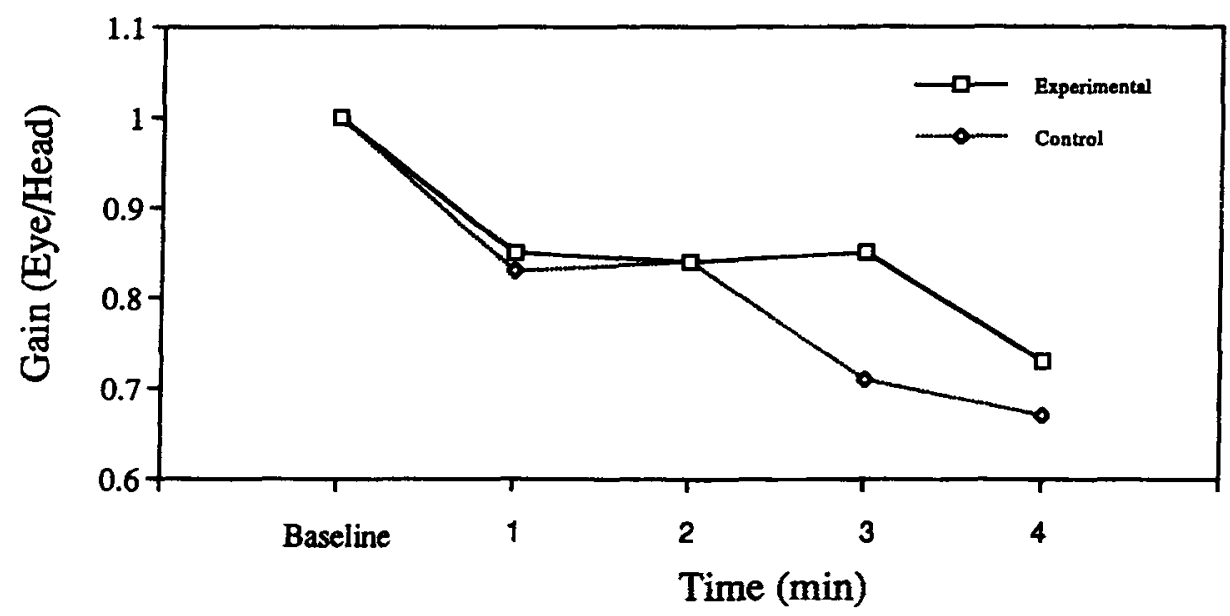

Figure 5. Experiment 2: Visual-vestibular gain, collapsed over four trials, as a function of exposure tíme for experimental (dual-adapted) and control groups. Baseline represents a normalized average of four preadaptation tests. Subsequent gains are evaluated relative to this measure. 
One possibility is that, for some reason, passive turning makes it easier to suppress the VOR. Another is that this result is due, in some way, to the fact that self-initiated head turning is inherently more variable than is externally controlled whole body rotation produced by a motor-driven rotating chair and that such variability interferes with the adaptive process. Furthermore, it should be noted that the total exposure period used here (4 min) was very short, as compared with the $30-60 \mathrm{~min}$ periods found in most previous research investigating the role of active interaction in adaptation (see, e.g., Held \& Hein, 1958). Perhaps the difference in VOR adaptation would have eventually favored the active condition if our exposure period had been extended.

There are a number of differences between the active and the passive conditions of the present experiment that might have accounted, in some way, for their differential outcomes with respect to adaptation and dual adaptation. First, our active condition involved only head rotation, whereas the passive rotation condition included the entire body. Thus, vestibulocolic reflexes would be involved only in the active condition. The generation of an efference copy during active head turning might also be important. Although the "traditional" role of efference in facilitating adaptation (see, e.g., Held \& Hein, 1958) was not confirmed here, it may have been an important reason that dual adaptation was limited to the active movement condition. The attentional state of the subject may also have differentiated the two conditions, since it is likely that subjects attempting to actively swing the head to the sound of the metronome were exerting more attention to their activities than were those sitting passively in a rotating chair. Finally, since the centers of rotation of the chair and of the head on the body were slightly different (although we endeavored to keep them as similar as possible), otolithic contributions probably differed slightly in the two conditions. Thus, the otoliths would have been stimulated because the inner ear vestibular apparatus translates slightly, the centers of rotation being not quite coincident with either of the otolith organs.

\section{GENERAL DISCUSSION}

\section{A Control Study}

It was pointed out to us by the reviewers of the initial version of this article that, owing to its unusual rapidity, what we considered to be VOR adaptation may merely have been evidence of a deliberate suppression of the VOR (albeit incomplete). Barr et al. (1976) demonstrated that human beings are capable of such suppression by simply imagining that they are fixating on a target that is moving with the head. If this alternative interpretation applies to our data, it would mean that we had not demonstrated dual adaptation of VOR, but merely that, with practice, subjects can get better at suppressing their VOR. One way to test the VOR suppression interpretation of our data is to interpose a cognitive task between the end of exposure to the altered target-head relationship and the final measure of VOR. The assumption here is that any tendency toward deliberate suppression of the VOR is transient and easily disrupted by an interfering task. Therefore, if a reduction of the VOR gain is still obtained after this interval, it may be concluded that our exposure condition was producing genuine VOR adaptation. We carried out a control study to test this hypothesis.

The exposure and VOR measurement protocol for the control study was identical to that used in Experiment 2, with the exception that, after the fourth and final VOR measurement, rotation of the chair was stopped, and the subjects were asked to count aloud backwards from 1,000 by threes for $3 \mathrm{~min}$. After this 3-min interval, the chair was restarted, and VOR gain measured once more. Six subjects participated in this control study, although one of them was excused for demonstrating eye movements that exceeded the range of our apparatus.

The average gain for the four exposure periods was $0.76(S D=0.11)$, which is in the adaptive direction and was statistically different from zero $(t=4.8, p<.01)$. The gain measured after the 3 -min cognitive task was $0.69(S D=0.09)$, also statistically different from zero $(t=7.5, p<.01)$. This reduction in gain was greater than that obtained immediately after the exposure period, although the difference between the two gains proved to be nonsignificant $(t=1.4, p=.23)$. Most important, the fact that the adaptive VOR gain persisted despite the interposed cognitive task is consistent with our contention that our exposure conditions produced genuine VOR adaptation rather than cognitive suppression.

\section{Conclusions}

Overall results and their interpretation. The combined results of Experiments 1 and 2 indicate that dual adaptation of the VOR can be elicited by repeated alternation between dysmetric and normal visual-vestibular environments, but only if bodily rotation is self-induced. Thus, although VOR adaptation occurred with passive rotation (Experiment 2), dual adaptation apparently did not. Furthermore, whether rotation was active or passive, no evidence of adaptive generalization was obtained, at least when tested by means of the 1.0 target/head gain that served here as the novel stimulus condition.

The apparent absence of dual adaptation with passive bodily rotation agrees with the results of Miles and Eighmy (1980) for the rhesus monkey. Lack of dual adaptation is also consistent with those mathematically based theoretical models (see, e.g., Ito, 1972) that, because they entail only one free parameter for VOR gain, do not include the possibility of storing previously exposed but presently inactive gains, which is an inherent characteristic of dual adaptation.

On the other hand, the results of the previously mentioned study by Shelhamer et al. (1992) appear to conflict with our results, as well as with those of Miles and Eighmy (1980), by demonstrating dual (or context-specific) VOR adaptation for subjects who, like those in the present Experiment 2 , were passively rotated in a motor-driven chair. 
However, a comparison between the discriminative (or switching) cues available to subjects in the present investigation and those provided by Shelhamer et al. may help to resolve this apparent contradiction. It will be recalled that observers in the latter study were exposed to one altered target/head gain while directing their eyes upward and a different gain while directing their eyes downward. The distinctly dissimilar sets of neural instructions (efference) associated with these two eye deviations probably served as a very salient cue for determining which of the two acquired VOR adaptations to use in a given situation. Clearly, no such efferent discriminative cue was available to the passively rotated observers in the present Experiment 2, perhaps explaining why they were apparently incapable of achieving dual adaptation.

The manner in which efference might serve as a discriminative cue for the dual adaptation obtained in the active head-turning condition (Experiment 1) is not as obvious as in the Shelhamer et al. (1992) study. That is, in Experiment 1, neural outflow was identical for the altered and normal gain conditions (i.e., a $0.45-\mathrm{Hz}$ oscillation rate in each case). Therefore, one must seek the discriminative cue for dual adaptation in this situation in the characteristics of the action-contingent visual feedback, or reafference, that differentiated the two gain conditions. In short, the distinct mismatch between efference and reafference that was present for the 0.5 target/head gain condition, but not for the normal $(0.0)$ gain condition, may have served as an effective discriminative cue for eliciting dual adaptation. Clearly, no such difference was present during passive whole body rotation (Experiment 2), since visual reafference, by definition, requires active movement. Thus, according to the present argument, the difference between the two gain conditions with respect to the discrepancy between vestibular stimulation and apparent concomitant motion, although sufficient to produce adaptation in the passive (as well as the active) condition, was insufficient for acquiring dual adaptation.

None of preceding speculations precludes afferent stimulation as a discriminative cue for dual adaptation in other contexts. Indeed, the common observation that wearers of spectacles are eventually able to don and doff their corrective lenses without experiencing the perceptual and behavioral problems that occurred initially is evidence of dual adaptation in which the feel of the frames (an afferent stimulus) is likely to be an important discriminative cue.

An alternative explanation for why active head turning led to dual adaptation whereas passive rotation did not is that self-initiated head turning may increase the subject's arousal level. As mentioned previously, this factor is known to facilitate VOR adaptation (see, e.g., Barr et al., 1976) and, thus, might have the same effect on dual adaptation.

In a very recent paper by Post and Welch (1998), dual adaptation, measured in terms of apparent concomitant motion, was produced in a situation in which the most salient discriminative cue was the rate of active head os- cillation $(0.25 \mathrm{~Hz}$ vs. $2.0 \mathrm{~Hz})$. On the basis of the reasoning presented above, this result is not surprising, given that this switching stimulus for dual adaptation included the distinctly different efferent instructions to the neck muscles required for the two head rotation rates. Furthermore, on the basis of the preceding arguments, it may be predicted that an attempt to repeat the Post and Welch Experiment with passive rotation will fail to produce dual adaptation, as it apparently did here.

Neurological implications. The observation of dual adaptation of the VOR may necessitate certain modifications of current neurological models. A synthesis of behavioral, neurological, and computer simulation data suggests that there are three primary neurological pathways by which VOR adaptation can occur (du Lac, Raymond, Sejnowski, \& Lisberger, 1995): (1) vestibular inputs to position-vestibular-pause (PVP) cells to extraocular motorneurons, (2) vestibular inputs to flocculus target neurons (FTN) to extraocular motorneurons, and (3) vestibular inputs to horizontal-gaze velocity purkinje (HGVP) cells to an inhibitory connection to the FTNs (no direct connection to extraocular motorneurons). Each of these structures (PVP, FTN, and HGVP) receives eye movement feedback through an efference copy of the eye movement motor commands.

The dynamics of the time course of the VOR suggests a different role for each of these pathways. Lisberger (1984) found that the first $5 \mathrm{msec}$ of VOR eye movement were unaffected by the current adapted VOR gain. This unmodifiable component can be attributed to the PVP pathway, since, of the three, this pathway is the only one with few enough synapses to account for the short $(14 \mathrm{msec})$ latency of the initial response. Indeed, it seems that this pathway accounts for baseline VOR response, as cell responses seem immune to changes in VOR gain per se (Lisberger, Pavelko, \& Broussard, 1994). Therefore, it seems unlikely that vestibular inputs to PVP cells are involved in adaptive changes. In contrast, the FTN pathway is clearly implicated in VOR gain adaptation. The estimated time course of activation through the pathway is $19 \mathrm{msec}$, which matches the latency of the modifiable component of the VOR response (du Lac et al., 1995). The firing rate of these neurons correlates well with changes in VOR gain, as an increase in adapted gain results in an increased firing among these neurons; conversely, a lowering of the gain results in a decreased firing. These results pertain even when the feedback effects of eye movements are controlled and strongly imply that vestibular inputs to FTN cells are at least partially responsible for adaptive changes in the VOR.

HGVP cells are also strongly implicated in VOR gain adaptation. The estimated latency for this pathway is $32 \mathrm{msec}$ (du Lac et al., 1995), implying that this cerebellar structure cannot be completely responsible for VOR gain changes (in contrast to Ito, 1972). However, the firing pattern of HGVP cells is consistent with changes in VOR adaptation levels. Since HGVP cells do not directly project to the extra-ocular motor neurons and, instead, 
serve to inhibit the FTN cells, an implication of HGVP cells in VOR adaptation would predict a lowering of activity when the gain is high and an increase in activity when the gain is low. Indeed, this is the case (Miles \& Eighmy, 1980).

However, in addition to the latency problem, there is another reason that adaptation cannot be completely in the cerebellum. Once the feedback effects of eye movements are controlled, the firing pattern of HGVP cells reverses; higher adapted gains result in more activity, lower in less activity. This somewhat paradoxical result merely implies that the HGVP cells or their inputs cannot be completely responsible for adaptation. According to computer simulations, HGVP cells show the expected pattern of response as long as inputs to FTN cells are also changed, and the changes to the inputs to the HGVP cells involve both temporal and magnitude dimensions (Lisberger, 1994).

The neural locus of VOR adaptation effects is often thought to be the flocculus of the cerebellum (Ito, Shiida, Yagi, \& Yamamoto, 1974), although some evidence suggests that recalibration occurs outside this structure and is merely transmitted through it (Lisberger \& Fuchs, 1977). In either case, no neural structure has yet been identified with the capacity to represent a recalibration signal on a long-term basis at the same time as another calibration is being implemented, which, of course, is precisely what dual adaptation of the VOR requires.

Adaptive generalization. The present investigation has demonstrated that VOR adaptation is more complicated than simply resetting an internal gain, since it is possible for the system to adapt to two different gains simultaneously. On the other hand, such dual adaptation does not appear to represent a generalized increase in the ability to adapt to other atypical target/head gains, since no "savings" in the form of adaptive generalization could be demonstrated when dual-adapted subjects were exposed to a novel gain (1.00). Perhaps this failure to find adaptive generalization is related to the finding that VOR adaptation is subject to a relatively steep generalization gradient when tested at head-turning frequencies differing from the one for which adaptation was initially acquired (Lisberger, Miles, \& Optican, 1983).

Implications for preflight adaptation training. The dual adaptability of the VOR has important implications for current attempts to provide astronauts with preflight adaptation training (PAT). In general, PAT entails using computer-controlled simulators to create and expose astronauts to the sensory and sensory-motor conflicts they will experience when they finally encounter the hypogravity of Earth orbit or on a trip to extraterrestrial bodies such as the Moon or Mars (see, e.g., Parker, Reschke, Ouyang, Arrott, \& Lichtenberg, 1986). Perhaps the most important of these conflicts are those involving the visualvestibular system, whose disruption in microgravity has been implicated in certain visual illusions, as well as space motion sickness (see, e.g., Crampton, 1990; Reason \& Brand, 1975). However, since practical considerations make it unlikely that PAT will be administered just before lift-off, it is important that astronauts be capable of retaining their adaptation for weeks or, perhaps, months beforehand, in the face of substantial intervening normal visual-vestibular experience. Dual adaptation is, by definition, an example of such long-lasting retention. The specific, albeit tentative, conclusions for PAT that may be drawn from the present results are that (1) the astronautin-training must be exposed to the simulated visualvestibular conflicts in an active manner and (2) on the basis of the present failure to obtain adaptive generalization, these conflicts must be as closely matched as possible to those they will actually experience in space.

The visual-vestibular mismatch in space, however, is primarily one of linear rather than rotary motion. That is, the rotary gains for visual images remain the same in space as on the ground, to the extent that they originate in the semicircular canals, since the canals are relatively unaffected by changes in gravity. On the other hand, the otolith organs do respond to gravity and are, therefore, greatly influenced by its absence. Lack of the normal gravity vector to sum with linear acceleration vectors in the otoliths means that every head displacement is accompanied by an otolith vector in a direction exactly opposite to the direction of acceleration. As a result, unadapted astronauts (or adapted astronauts who have just returned to Earth) feel that their heads are tilting with every translational movement (see, e.g., Parker et al., 1986). Therefore, for the most direct application to the problems of weightlessness during manned space flight, an analysis of vestibular plasticity of the sort performed here should be carried out with linear acceleration. Such an analysis is currently underway in our laboratory.

\section{REFERENCES}

Baker, J. F., Perlmutter, S., Peterson, B., Rude, S., \& Robinson, F. R. (1987). Simultaneous opposing adaptive changes in cat vestibuloocular reflex direction for two body orientations. Experimental Brain Research, 69, 220-224.

BARNES, G. R. (1993). Visual-vestibular interaction in the control of head and eye movement: The role of visual feedback and predictive mechanisms. Progress in Neurobiology, 41, 435-472.

Barr, C. C., Schultheis, L. W., \& Robinson, D. A. (1976). Voluntary non-visual control of the human vestibulo-ocular reflex. Acta OtoLaryngologica, 81, 365-375.

Collewijn, H., Martins, A. J., \& Steinman, R. M. (1981). The timecourse of adaptation of human compensatory eye movements. In L. Maffei (Ed.), Psychopathology of the human visual system. Documenta Opthamologica Proceedings Series, 30, 123-133.

Collewijn, H., Martins, A. J., \& Steinman, R. M. (1983). Compensatory eye movements during active and passive head movements: Fast adaptation to changes in visual magnification. Journal of Physiology, 340, 259-286.

CRAMPTON, G. A. (1990). Motion and space sickness. Boca Raton, FL: CRC Press.

du Lac, S., Raymond, J. L., Sejnowski, T.J., \& Lisberger, S. G. (1995). Learning and memory in the vestibulo-ocular reflex. Anmual Review of Neuroscience, 18, 409-441.

HELD, R., \& HeIN, A. (1958). Adaptation to disarranged hand-eye coordination contingent upon reafferent stimulation. Perceptual \& Motor Skills, 8, 87-90.

Howard, I. P. (1982). Human visual orientation. New York: Wiley. 
ITo, M. (1972). Neural design of the cerebellar motor control system. Brain Research, 40, 81-84.

Ito, M., Shidda, T., Yagi, N., Yamamoto, M. (1974). Visual influence on rabbit's horizontal vestibulo-ocular reflex that presumably is effected via the cerebellar flocculus. Brain Research, 65, 170-174.

Jell, R. M., Stockwell, C. W., Turnispeed, G. T., \& Guedry, F. E. (1988). The influence of active versus passive head oscillation, and mental set on the human vestibulo-ocular reflex. Aviation, Space, \& Environmental Medicine, 59, 1061-1065.

LISBERGER, S. G. (1984). The latency of pathways containing the site of motor learning in the vestibulo-ocular reflex. Science, 225, 74-76.

LISBERGER, S. G. (1994). Neural basis for motor learning in the vestibuloocular reflex of primates: III. Computational and behavioral analysis of the sites of learning. Journal of Neurophysiology, 72, 974-999.

Lisberger, S. G., \& Fuchs, A. F. (1977). Control of gaze by brain stem neurons. In R. Baker \& A. Bertoz (Eds.), Neuroscience developments (pp. 381-389). Amsterdam: Elsevier.

Lisberger, S. G., Miles, F. A., \& Optican, L. M. (1983). Frequencyselective adaptation: Evidence for channels in the vestibulo-ocular reflex? Journal of Neuroscience, 3, 1234-1244.

Lisberger, S. G., Pavelko, T. A., \& Broussard, D. M. (1994). Neural basis for motor learning in the vestibulo-ocular reflex of primates: I. Changes in the responses of brainstem neurons. Journal of Neurophysiology, 72, 909-927.

MELVILL JONES, G. (1985). Adaptive modulation of VOR parameters by vision. In A. Berthoz \& G. Melvill Jones (Eds.), Adaptive mechanisms in gaze control (pp. 21-50). New York: Elsevier.

Miles, F. A., \& EIGHMY, B. B. (1980). Long-term adaptive changes in primate vestibuloocular reflex: I. Behavioral observations. Journal of Neurophysiology, 43, 1406-1425.

Parker, D. E., Reschke, M. F., Ouyang, L., Arrott, A. P., \& LichtENBERG, B. K. (1986). Vestibulo-ocular reflex changes following weightlessness and preflight adaptation training. In E. Keller \& D. Zee (Eds.), Adaptive processes in visual and oculomotor control systems (pp. 103-109). Oxford: Pergamon.

Post, R. B., \& LoTT, L. A. ( 1992). The relationship between vestibuloocular reflex plasticity and changes in apparent concomitant motion. Vision Research, 32, 89-96.

POST, R. B., \& WELCH, R. B. (1998). Dual adaptation of apparent con- comitant motion contingent on head rotation frequency. Perception \& Psychophysics, 60, 821-825.

Reason, J. T., \& BRAND, J. J. (1975). Motion sickness. New York: Academic Press.

RONNE, H. (1923). Mouvements apparents, produits a la vision par verres de lunettes, et la correction de ces mouvements par les canaux semicirculaires. Acta oto-laryngology, 5, 108-110.

Shelhamer, M., Robinson, D. A., \& Tan, H. S. (1992). Contextspecific adaptation of the gain of the vestibulo-ocular reflex in humans. Journal of Vestibular Research, 2, 89-96.

TIETZ, J. D., \& GoGEL, W. C. (1978). Adaptation to apparent concomitant motion in the absence of physical or retinal motion. Bulletin of the Psychonomic Society, 12, 1-4.

WELCH, R. B. (1978). Perceptual modification: Adapting to altered sensory environments. New York: Academic Press.

Welch, R. B., Bridgeman, B., Anand, S., \& Browman, K. E. (1993). Alternating prism exposure causes dual adaptation and generalization to a novel displacement. Perception \& Psychophysics, 54, 195-204.

\section{NOTE}

1. The term eye/head gain is to be distinguished from target/head gain. The former describes what the eyes do when the head rotates; it serves as the dependent variable of the present experiments and is used here synonymously with VOR gain. An eye/head gain of 1.0 indicates that the eyes have turned in one direction just as far as the head has turned in the opposite direction and have, thus, completely compensated for the head rotation. In contrast, the term target/head gain refers to what a visual target is caused to do by the investigator when the subject's head turns. Thus, it can be thought of as an independent variable. Here, a ratio of 1.0 signifies that a visual target has been physically moved by the same amount and in the same direction as that in which the head has turned and, thus, should initially appear to be moving along with the head. Perception of a stable visual field requires a target/head gain of approximately 0.0 .

(Manuscript received February 18, 1997; revision accepted for publication November 12, 1997.) 